\title{
Free Vibration Analysis of Rotating Functionally-Graded Cantilever Beams
}

\author{
M. N. V. Ramesh \\ Department of Mechanical Engineering, Nalla Malla Reddy Engineering College, Hyderabad-500088, India
}

\begin{abstract}
N. Mohan Rao
Department of Mechanical Engineering, JNTUK College of Engineering Vizianagaram, Vizianagaram-535003, India
\end{abstract}

(Received 23 May 2012; accepted 20 August 2013)

The increasing needs of the industry involved in development of components for aerospace and power sector demand the engineering community to develop new concepts and strategies to improve the functional requirements of structures and to enhance the strength of materials. This is particularly essential in the cases of rotating beams that are subjected to severe vibration under large pressure loadings, high rotating accelerations, centrifugal forces, geometric stiffening, etc. A theoretical investigation of the free vibration characteristics of rotating cantilever beams, made of a functionally-graded material (FGM) consisting of metal and alumina, is presented in this study. It was assumed that the material properties of the FGM beam were symmetric, but varied continuously in the thickness direction from the core at the mid section to the outer surfaces, according to a power-law relation. Equations of motion were derived from a modelling method, which employed the hybrid deformation variable. The natural frequencies were determined using the Rayleigh-Ritz method. The effect of parameters such as the power law index, the hub radius, and the rotational speed on the natural frequencies of functionally-graded rotating cantilever beams were examined through numerical studies and then compared with the numerical results reported in earlier works.

\section{NOMENCLATURE}

$\vec{a}^{P}$
$A$
$b$
$E_{(z)}$
$h$
$\hat{l}, \hat{j}, \hat{k}$
$J_{11}^{E}$
$J_{11}^{\rho}$
$J_{22, y y}^{E}, J_{22, z z}^{E}$

$L$

$n$

$\vec{P}$

$P_{(z)}$

$P_{(m)}$

$P_{(c)}$

$q_{1 i}, q_{2 i}, q_{3 i}$

$r$

$\rho_{(z)}$

$s$

$T$

$u, v, w$

hub
Acceleration vector of the generic point $\mathrm{P}$

Cross-sectional area of the beam

Width of the beam

Youngs modulus

Total thickness of the beam

Orthogonal unit vectors fixed to the rigid

Axial rigidity of the beam

Mass density per unit length

Flexural rigidities of the functionallygraded beam

Length of the beam

Power law index

Vector from point $\mathrm{O}$ to $P_{0}$

Effective material property

Metallic material property

Ceramic material property

Generalized co-ordinates

Radius of the rigid frame

Mass density per unit volume

Arc length stretch of the neutral axis

Reference period

Cartesian variables in the directions of $\hat{i}, \hat{j}$, and, $\hat{k}$
$U$

$\vec{v}^{O} \quad$ Velocity of point $\mathrm{O}$

$\vec{v}^{P} \quad$ Velocity vector of the generic point $\mathrm{P}$

$x \quad$ Spatial variable

$\gamma \quad$ Ratio of the angular speed of the beam to

the reference angular speed

$\delta \quad$ Hub radius ratio

$\Theta \quad$ Constant column matrix characterizing the deflection shape for synchronous motion

$\mu_{1}, \mu_{2}, \mu_{3} \quad$ Number of assumed modes corresponding

to $q_{1 i}, q_{2 i}$, and $q_{3 i}$

$\tau \quad$ Dimensionless time

$\phi_{1 j}, \phi_{2 j}, \phi_{3 j}$ Modal functions for $s, v$ and $w$

$\vec{\omega}^{A} \quad$ Angular velocity of the frame A

$\Omega \quad$ Angular speed of the rigid hub

(') Partial derivative of the symbol with respect to the integral domain variable

(") Second derivative of the symbol with respect to the integral domain variable

\section{INTRODUCTION}

Functionally-graded materials are special composites whose properties change spatially in one or more directions. Functionally-graded structures are being widely applied in extremely high temperature environments like those occurring 